\title{
Transatlantica
}

Revue d'études américaines. American Studies Journal

\section{Christophe Lebold, Leonard Cohen : L'Homme qui voyait tomber les anges}

Camion Blanc, 2013

\section{Vincent Dussol}

\section{(2) OpenEdition}

\section{Journals}

Édition électronique

URL : https://journals.openedition.org/transatlantica/6591

DOI : 10.4000/transatlantica.6591

ISSN : 1765-2766

Éditeur

Association française d'Etudes Américaines (AFEA)

Édition imprimée

Date de publication : 31 décembre 2013

Référence électronique

Vincent Dussol, «Christophe Lebold, Leonard Cohen : L'Homme qui voyait tomber les anges », Transatlantica [En ligne], 2 | 2013, mis en ligne le 30 avril 2014, consulté le 09 février 2023. URL : http:// journals.openedition.org/transatlantica/6591; DOI : https://doi.org/10.4000/transatlantica.6591

Ce document a été généré automatiquement le 9 février 2023

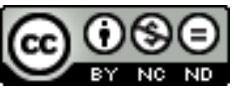

Creative Commons - Attribution - Pas d'Utilisation Commerciale - Pas de Modification 4.0 International - CC BY-NC-ND 4.0

https://creativecommons.org/licenses/by-nc-nd/4.0/ 


\section{Christophe Lebold, Leonard Cohen: L'Homme qui voyait tomber les anges}

Camion Blanc, 2013

Vincent Dussol

\section{RÉFÉRENCE}

LEBOLD, Christophe, Leonard Cohen : L'Homme qui voyait tomber les anges, n.p., Camion Blanc, 2013, 720 pages, ISBN : 978-2-35779-397-2, $36 €$

1 Le titre de ce fort volume de sept cents pages illustre parfaitement le point de vue exposé au deuxième chapitre. Aucun portrait, aucun récit de vie n'a d'intérêt sans " "théorie", (c'est-à-dire un point de vue). [...] S'en tenir strictement aux faits est impossible, ne serait-ce que parce que les faits sont insaisissables et opaques» (56-57). C'est la représentation, qui est interprétation, qui peut le mieux restituer une vérité. La leçon de Henry James, en somme, dans sa nouvelle « The Real Thing ».

Les treize chapitres du livre, qui suivent de façon chronologique les grandes étapes de la vie de Leonard Cohen jusqu'en 2013, sont précédés d'un «Prologue » qui propose trois points d'entrée dans l'œuvre dont le lecteur, dit Lebold, usera à sa guise. Le premier fait écho au titre du livre et propose une lecture de l'œuvre et de la vie de Cohen à partir du couple gravité-légèreté. L'auteur montre comment celui-ci est opératoire tant pour décrire les évolutions de la voix de l'artiste que pour rendre compte de son rapport, fait de profondeur et d'humour résigné, aux chutes inévitables de la vie, des plus physiques aux plus métaphysiques. La place de la culture juive dans l'œuvre de Cohen constitue le second point d'entrée. De façon très convaincante, le biographe unifie ce champ autour de quelques notions (errance, combat avec l'ange, exil) et suggère au passage que pour son sujet, le nom - «un kohen [...] désigne le prêtre » (35) -, a pu faire destin. S'ébauche ici une mise en communication de la pensée juive avec le bouddhisme zen, deux spiritualités qui n'auront cessé de se nourrir mutuellement chez Cohen. Le thème cohénien de l'hôtel amène par exemple Lebold à 
s'arrêter sur la poétique de ce lieu d'hospitalité anonyme pour le pèlerin et l'errant moderne. Troisième clé : ce que le biographe regroupe sous l'en-tête " La Métaphysique des Cœurs Brisés ». Il montre comment peut se penser le passage entre deux multiplicités chez son sujet : l'amour et le chant. Entre foi toujours renaissante en la rédemption mystique par le premier et réparation par les étincelles de lumière du second, se déploie l'éventail de la mélancolie qui chez le poète-chanteur canadien est davantage motrice que paralysante. Là encore, c'est la spiritualité juive qui fournit à l'auteur un regard éclairant sur la question.

3 Quel que soit le point d'entrée choisi, on est saisi d'emblée par le souci du biographe de maintenir constamment en tension des pôles très différents de l'objet analysé. L'écueil des vieilles dichotomies (la vie et l'œuvre, l'auteur-compositeur et l'interprète) est ainsi élégamment dépassé et on se laisse volontiers prendre par l'exposé de la consistance d'une vie qui fait univers.

4 Le premier chapitre fait découvrir le terreau où s'enracine la vocation de Leonard Cohen à être qui il sera. C'est l'histoire d'une enfance heureuse dans une famille juive aisée de Montréal qui possède entre autres biens une usine de confection - où l'on comprend que l'élégance raffinée de Leonard lui vient de sa culture familiale. C'est aussi le récit de l'éveil artistique qui, dans son cas, passe par la scène poétique, héritière du Modernisme, du Montréal des années 1950. Alors qu'il aborde le portrait de son personnage, Lebold prend le temps d'une belle méditation sur le visage dont la pertinence est riche d'échos dans l'œuvre de Cohen même, exemple parfait d'une démarche toujours réfléchie de l'auteur. Autre originalité de ce premier chapitre, le rapide survol, en sept actes, de l'histoire qui va suivre. On ne peut qu'apprécier cette aide supplémentaire au lecteur qui peut ainsi commodément se reporter à ce squelette chronologique, mais il faut y voir surtout la réaffirmation de la primauté de l'éclairage par rapport aux faits. "L'histoire donc [...] on la connaît » (63). Suit l'analyse des moments charnières de la jeunesse : la découverte du corps des femmes dont le culte cohénien ne le cède en rien à celui développé par la grande mystique courtoise, celle de la voix de Ray Charles, le rôle structurant de la religion juive chez Leonard que, dans un raccourci dont il a le secret, Lebold associe à la préférence de son sujet pour les métriques régulières en poésie, les costumes bien coupés et la recherche des postures parfaites dans le zen.

5 Le second chapitre, qui s'arrête à l'orée des années 1960, débute par une vision en raccourci de la décennie précédente. On voit sur ce fond Leonard commencer à tracer sa propre route : il est représentatif de son époque tout en s'en démarquant. C'est vrai pour sa poésie : bien que le titre de son premier recueil - Let Us Compare Mythologies puisse faire penser à l'influence Moderniste du groupe de poètes montréalais au sein duquel il fait ses armes, le jeune homme prend ses distances avec l'impersonnalité et bouscule les limites de ce qu'on appellera plus tard le politiquement correct. Mêmes décalage et différence par rapport à l'esprit du temps dans ses choix de vie: un peu aventurier et en avance sur la pastorale hippie, il s'installe sur une île grecque, va brièvement au contact de la révolution à $\mathrm{Cuba}$, mais n'en revient pas politisé: contrairement à un Dylan, Cohen est un homme du temps long. Est-ce pour cela qu'il trouve naturellement sa place dans la communauté d'artistes venus du nord s'installer à Hydra au plus près des sources de la poésie ? Lebold croque à merveille le lieu et le moment : nous sommes entre More de Barbet Schroeder (1969) et le Henry Miller du Colosse de Maroussi. 
6 Leonard Cohen fut d'abord écrivain avant de venir à la chanson. S'il l'avait vaguement su, le lecteur de ce livre ne l'oubliera plus. Il y a dans les pages de critique génétique poétique du troisième chapitre une contamination heureuse du biographe par le biographié. "Il y est question de papillons et de guêpes, du vent qui joue dans les citronniers et des fourmis qui se promènent autour des pieds nus de sa muse, du bruit de cloches aussi qui sanctifie l'espace" (151). Qu'un poème soit la résultante de conditions objectives ne fait plus aucun doute. Mais ce que démontrent aussi tous les faits rassemblés par Lebold, c'est le sens quasi-whitmanien de l'auto-publicité dont fait preuve Cohen dans sa première carrière de poète. Romans et recueils s'enchaînent entre 1961 et 1966. L'apprenti-star façonne son image, tourne d'un bout à l'autre du Canada pour donner des lectures, en découd avec le provincialisme pudibond de son pays en faisant volontiers rimer obscénité et sainteté. Il se sait poète dans une "société du spectacle " (158) et joue avec succès de tous les canaux pour se faire une place au soleil. Et lorsqu'en 1966, il s'affiche chanteur, il est déjà rompu à la dimension publique du métier.

7 Les « enfances du héros » maintenant trentenaire se poursuivent au chapitre 4, à New York cette fois, abordée en un panorama multimédia qui permet ensuite à Lebold de se déplacer souplement d'hôtel en hôtel, suivant Leonard de rencontre en rencontre ; et il en fait d'inoubliables (Judy Collins, Janis Joplin, Nico) qui resserrent pour le lecteur le tissu de sa vision de l'époque tout en en plaçant les astres à plus exacte distance l'un de l'autre (la rue épique pour Dylan, la chambre lyrique pour Cohen etc.). Tels les ascenseurs du Chelsea Hotel, le biographe brouille les étages en métaphorisant la vie du biographié, cependant qu'il insiste sur le poids de vie, la « fonction heuristique » (221) des métaphores: ainsi convoque-t-il la théologie et l'angélologie pour une microlecture de "Suzanne »; il déplie aussi la question de la nécessité de l'image de l'ange pour le poète contemporain - verbal ou visuel - de Wallace Stevens à Wim Wenders en passant par Rilke. Parallèlement nous suivons pas à pas l'insertion de Leonard sur la scène folk. Adepte de la prolepse, Lebold crée constamment des surplombs temporels, rebrousse chemin, joue avec son lecteur qui attend, bien sûr, la montée sur la grande scène du personnage pour lequel il a acheté le livre... En attendant, voici une autre mise en appétit par une interprétation de "Sisters of Mercy" ou encore une "théorie générale de l'amour cohénien", nourrie de références à la kabbale et à Michel de Certeau, à propos de la rencontre du poète avec la chanteuse du Velvet Underground. Disons ici que les tentatives de mise en cohérence poétique par l'auteur des amours de Leonard ne seront pas toutes aussi réussies et convaincantes que celle-ci, sûrement du fait de leur répétition. Cohen a trouvé en Christophe Lebold son infatigable apologiste, mais tous les jeux de l'amour ne valent pas la chandelle du critique.

8 Avec le chapitre 5, nous sommes en 1967-1968: on aborde la carrière de musicien de Leonard. Lebold nous fait vivre toute la force de l'événement, artistique et médiatique, que constitue ce premier disque qu'est Songs of Leonard Cohen qui, par sa forme d'intemporalité et son sujet central, propulse son auteur dans le rôle de voix du moment, à la fois troubadour et grand-prêtre de l'amour. Où l'on voit le poète peaufiner son image en creusant l'érotique de la métaphysique et vice-versa.

9 Il faut sûrement se défaire du cliché qui associe Cohen au "soleil noir de la mélancolie ». On apprend dans le livre comment, même dans ses périodes de dépression clinique, la distance de l'humour et de l'ironie n'a jamais quitté l'auteur de «So Long, Marianne ». Mais très intéressantes sont ces pages où est analysé plus en 
détail ce qui différenciait le nouveau venu, « adepte résolu du mode mineur » (286), de "Janis, Jim et Jimi », occupés à écrire "le grand récit rock [...] sur le mode épique " (285) ; or, l'épopée, discours guerrier hyperbolique, mène souvent à de tristes fins. Et Lebold de proposer pour Cohen une ascendance de rockeurs alternatifs de l'obscurité lumineuse qui part de Dante à Sylvia Plath et passe par Saint Jean de la Croix ! Le texte du sixième chapitre couvre la période des opus 2 et 3 du chanteur. Sans avoir recours à un vocabulaire musical trop technique, nous y est décrit ce qui fait la spécificité et la réussite de la musique de Leonard Cohen lorsqu'elle rencontre la fine fleur de Nashville en la personne d'un producteur qui comprend ce qui la servira le mieux : Bob Johnston, sorte d'anti-Phil Spector, qui sait la vertu du dépouillement. La country a toujours porté en elle la splendide sobriété des American Recordings de Johnny Cash et c'est celle-ci qui sied au propos de Cohen sur son deuxième disque. Songs from a Room est lu par Lebold comme une interrogation métaphysique sur la liberté qui, malgré l'indirection poétique, fait mouche en pleine guerre du Vietnam en célébrant par exemple la beauté de la défaite dans «Bird on The Wire » ou celle de la résistance à l'occupant dans « The Partisan ». Le public a bien senti qu'un puissant refus s'exprime là. Le biographe va plus loin, parle du NON cohénien comme d'un "pessimisme tonique " (305), libérateur, et fait le lien avec la légèreté du zen qui va bientôt saisir Leonard. Ces premières années d'immense popularité sont ponctuées par les tournées de concerts. Un passage obligé est donc celui du portrait de l'artiste sur scène. Que dire sinon que seule manque la musique à cette évocation d'un concert de 1970 qui veut les dire tous, reconstitution sur la page de la chaleur communielle, de l'intériorité qui semble avoir été la marque de nombre de ces soirs, y compris en clôture du festival de l'île de Wight, devant 600000 personnes. Le dernier grand jalon de ces années à la charnière des deux décennies, c'est Songs of Love and Hate (1971). À lire l'intertitre de la section que lui consacre Lebold «Manuel Anti-Hippie " - et les pages qui suivent, on est tenté de faire un parallèle entre deux gestes d'indépendance : celui de Dylan qui reversait en 1965, les barrières du folk à Newport - renonçant par là à l'allégeance de son premier public - et celui de Cohen, qui, tout juste passée la crête de la vague du peace and love renvoie les amoureux de l'amour à leurs naïvetés. Toujours attentif à la double nature de l'objet, le critique gratifie son lecteur d'analyses littéraires et musicales des chansons les plus marquantes.

10 Au fil des ans, les événements tendent, pour le commun des mortels, à se raréfier, à s'amenuiser, en tout cas à se ressembler. Il faut pourtant sept chapitres à Lebold pour rendre justice aux quarante années qui suivent. Le treizième et dernier, qui va de 2001 à 2013, au terme duquel nous quittons le protagoniste maintenant âgé de soixante dixneuf ans, occupe malgré tout cinquante-cinq pages. Le biographe traduit ainsi un fait d'expérience avec les authentiques artistes à longévité artistique exceptionnelle : ils proposent jusque dans leur grand âge de nouvelles portes d'entrée possibles dans leur œuvre et il peut très bien s'envisager de découvrir le premier Cohen en partant du dernier en date. Voir, ici encore, Henry James et tant d'autres. C'est tout le sens d'une entreprise comme le présent récit de vie que de rendre tangible la flamme ininterrompue de création.

11 Elle continue de s'alimenter à plusieurs sources: c'est ce qu'on retient de cette deuxième moitié du livre. Ces différentes nourritures de l'œuvre abondante, ponctuée de nouveaux sommets et qui continue de se construire, sont autant d'occasions de digressions éclairantes : sur la pratique du kôan et plus généralement le bouddhisme zen rinzaï qui prend une importance croissante dans la vie de Leonard; sur une facette 
inédite de la guerre du Kippour (1973) durant laquelle, pendant les trois semaines du conflit, il met son talent au service du "divertissement des troupes» (395) sur le théâtre des opérations, ce qui relance une inspiration en panne; sur la tradition hébraïque qui soutient Cohen dans les dures épreuves du cœur et à laquelle il emprunte des formes littéraires - pour preuve éclatante, voir le Book of Mercy (1984); sur la technologie musicale avec l'apparition et le développement du synthétiseur dans lequel Leonard verra le véhicule idéal de la distance ironique, un garant contre les excès d'expressivité - Lebold parle d'un progressif «virage électro » (497); sur la forme du clip vidéo ou encore le situationnisme. Ici un inoubliable portrait en situation du producteur Phil Spector, là le récit très enlevé de la rencontre entre Cohen et Dylan en 1986 dans un bar de la Rue d'Alésia, ailleurs encore, ce qui ne peut que devenir la référence en matière de description de la voix cohénienne; et, au fil des tournées qui s'enchaînent, des masques qui se renouvellent, le spectacle du talent attelé aux stratégies marketing et aux formules musicales choisies. Course de grand fond et résilience sans borne! Route et routines mais la foulée du biographe reste alerte pour cou(v)rir le marathon de cette vie.

12 Les décisions esthétiques de Lebold pour son propre livre contribuent grandement à l'attrait de l'ensemble. Il cite les poèmes, en intervalle double, dans leur version originale et en donne la traduction en note de bas de page. Plutôt que de sacrifier à la tradition du cahier de photos, il a préféré enrichir le fil de son texte d'une iconographie abondante - photographies de Cohen au travail (sur scène ou à sa table d'écriture) ou bien prenant la pose, clichés des lieux essentiels, photogrammes de clips, dessins de l'artiste (autoportraits grotesques en particulier), pochettes de disques ou couvertures de livres : ces repos pour l'œil ne sont pratiquement jamais purement illustratifs ; ils continuent le récit autrement.

13 Si l'auteur refuse de prétendre à l'objectivité - un fantasme qui conduit à ces biographies désincarnées à force d'information - le lecteur n'aura pas manqué d'être impressionné par la connaissance encyclopédique démontrée par Lebold de la documentation disponible sur son sujet. Constamment des références croisées de tous ordres (filmiques, journalistiques, littéraires), un kaléidoscope de plans d'ensemble virages artistiques, tableaux d'une époque, grandes influences - et de plans rapprochés - sur telle femme aimée, tel lieu habité, telle composition, tel concert - de la constellation Cohen, seront venus étayer la «théorie » proposée. La finition de l'objet aurait pu être meilleure encore: on regrette les assez nombreuses coquilles et les quelques titres égarés en bas de page. Il n'empêche : le livre engage à la lecture et en approchant de la fin de l'ouvrage, on a surtout le sentiment de tenir là une manière de synthèse idéale entre le vif et l'archive, entre le très savant et la passion. Geste symbolique de cet équilibre: la sortie fugitive de la tête de derrière le rideau que s'autorise tardivement le biographe - «Un ange était passé et moi je poursuivais cet ange à travers l'Europe " (663). Outre sa ou ses "théories ", Lebold se sera cependant manifesté autrement tout au long du livre. Car ce travail est aussi un trésor: la bibliographie "style thèse» le prouve. Il aura visiblement été pour son auteur l'occasion de mettre en récit un florilège de citations philosophiques, de fragments de poèmes, de réflexions très abouties, de jeux de mots jubilatoires et justes qui l'accompagnent dans son quotidien et se sont lentement intégrés à sa vision de la vie et du monde. Il aura pu enfin les livrer ici en les imbriquant à son sujet. Conformément à l'adage de Nietzsche qu'il cite à deux reprises au moins, il sera, par ce livre, sans doute un peu plus devenu ce qu'il est. Il serait réducteur, bien que tentant, de dire que c'est 
l'esprit du rock qui a soufflé là, pour dessiner une nouvelle manière d'écriture de recherche où vibration rime avec érudition. Car cette summa coheniana dans la langue de Molière apparaît aussi comme la résultante autant que la récompense d'un rapport très privilégié entre l'auteur et compositeur canadien et le public français. Souhaitonslui le vaste lectorat qu'elle mérite!

\section{AUTEURS}

\section{VINCENT DUSSOL}

Université Paul Valéry, Montpellier 3 